\title{
Performance of Downlink Group-Orthogonal Multicarrier Systems
}

\author{
Felip Riera-Palou, Guillem Femenias, and Jaume Ramis \\ Dept. of Mathematics and Informatics \\ University of the Balearic Islands \\ 07122 Palma de Mallorca, Spain \\ \{felip.riera, guillem.femenias, jaume.ramis\}@uib.es
}

\begin{abstract}
Group-orthogonal multi-carrier code division multiple access (GO-MC-CDMA) has recently been proposed as a promising technique for the uplink segment of wireless systems. In this paper we propose and analyze a related scheme, group-orthogonal multi-carrier code division multiplexing (GO-MC-CDM), suitable for the downlink segment. The proposed receiver is shown to offer a similar bit error rate (BER) performance as the downlink version of GO-MC-CDMA at a fraction of its computational complexity. An analytical expression for the BER when using maximum likelihood (ML) detection is derived providing valuable insight into the parameters affecting the system performance and providing a basis for its optimization. Simulation results using parameters and channel models aiming at the next generation of wireless systems are provided confirming the analytically derived results.
\end{abstract}

Keywords: MC-CDMA, downlink, multi-symbol detection, rotated spreading.

\section{Introduction}

Multi-carrier code division multiple access (MC-CDMA) [1] can be seen as a specific case (see 2 for other possibilities) of the combination of two complementary techniques: code division multiple access (CDMA) and orthogonal frequency division multiplexing (OFDM). On one hand, CDMA multiplexes users by means of a user-specific spreading code allowing them to simultaneously use the same frequency spectrum. The properties of these codes (e.g. orthogonality) make user separation at the receiver possible. On the other hand, OFDM is a block transmission scheme where the incoming user symbols are grouped, serial-toparallel $(\mathrm{S} / \mathrm{P})$ converted and modulated onto different subcarriers. Choosing the subcarriers to be orthogonal allows the group of symbols to be transmitted in parallel without interference. The $\mathrm{S} / \mathrm{P}$ conversion permits the transmission rate to be reduced to a fraction of the original user rate combating in this way the frequency selectivity of the channel. The attractive features derived from the CDMA-OFDM combination makes MC-CDMA a firm candidate for the next generation of wireless systems [3]. Typically, multiuser detection (MUD) based on linear or non-linear processing is employed in the uplink due to its superior 
performance and the lack of tight computational constraints in the base stations [3. In the downlink, single-user detection is usually preferred owing to its lower computational cost.

Group-orthogonal MC-CDMA (GO-MC-CDMA) has been recently proposed in [4] as an attractive alternative for the uplink segment. It can be seen as the combination of MC-CDMA and orthogonal frequency division multiple access (OFDMA). The main idea behind GO-MC-CDMA is to partition the available (orthogonal) subcarriers into (orthogonal) groups and distribute users among the groups. The main advantage of this system is that each group functions as an independent MC-CDMA system with a smaller number of users making the use of maximum likelihood multiuser detection (ML-MUD) within each group feasible.

As with MC-CDMA, GO-MC-CDMA can in principle also be used for the downlink. The mobile user can then employ a detector targeting only the subcarriers forming the groups where his symbols are being transmitted. Notice that in the usual case where several symbols in parallel are transmitted for each user, the receiver will need a separate detector for each required group. As in the uplink, and depending on the resources available at the receiver, each group-wise detector can be single-user or multiuser 3. This latter case can be considered as rather inefficient since all the detected symbols in a group but one will be discarded as they belong to other active users in the network. A more appropriate solution consists of multiplexing all the (parallel) symbols from a given user in the same group similar to the OFDM code-division multiplexing (OFDM-CDM) scheme proposed in [5] hence the name of our proposal, group-orthogonal multicarrier code-division multiplexing (GO-MC-CDM). In contrast with (downlink) GO-MC-CDMA, in the proposed system the mobile user needs only targeting one single group which contains all the useful information, making multisymbol (rather than single symbol) detection more adequate as it can achieve the same performance as the multiuser counterpart (i.e. GO-MC-CDMA) at a fraction of its complexity.

In this paper we first present the architecture of the GO-MC-CDM and derive an analytical expression for the BER when employing maximum likelihood multisymbol detection (ML-MSD). Relevant parameters affecting the performance are identified allowing some design decisions to be optimally made. Simulation results are then provided, using typical parameters currently under discussion for the next generation of wireless systems, which illustrate the performance of GO-MC-CDM and serve also to validate the analytical results. We note that the analysis presented in this paper would also be valid for the downlink GO-MCCDMA, although as pointed out before, if multiple symbols are transmitted in parallel, then a separate ML detector would be required for each group.

\section{System Model for GO-MC-CDM}

This paper focuses on the (synchronous) downlink (base to mobile) of a multicarrier system with $N_{\text {total }}$ subcarriers serving $K_{\text {total }}$ users. Similarly to 
GO-MC-CDMA, the total number of subcarriers is partitioned into $N_{g}=N_{\text {total }} / N$ groups where $N$ is the number of subcarriers per group. Each active user in the system has exclusive use of the subcarriers forming a group. Due to the orthogonality among groups, multiuser interference is completely eliminated and therefore only self interference (i.e. inter symbol) should be addressed. Notice that $N$ is the parameter balancing the capacity of the system (number of users) and the maximum number of parallel symbols each user can transmit. Since groups are independent of each other, all the modeling and analysis can be performed on a single group which, to all effects, resembles the OFDM-CDM system proposed in $[5$.

The block diagram for the base station transmitter corresponding to user $k$ is shown in Fig. 1. At (discrete) time instant $n$, a block of $S$ successive data symbols $\mathbf{a}^{k}(n)=\left[\begin{array}{lll}a_{0}^{k} & a_{1}^{k} \ldots a_{S-1}^{k}\end{array}\right]^{T}$ with each symbol drawn from an M-ary complex-valued symbol constellation (e.g., M-QAM or M-PSK) and satisfying $E\left[\left|a_{s}^{k}\right|^{2}\right]=1$, is first serial-to-parallel converted. Each symbol $a_{p}^{k}$ is then multiplied by a different spreading code of the form $\mathbf{c}^{s}=\left[\begin{array}{lll}c_{0}^{s} & c_{1}^{s} \ldots c_{N-1}^{s}\end{array}\right]^{T}$ with $E\left[\left|c_{i}^{s}\right|^{2}\right]=1 / N$. For later convenience, we define now the $N \times P$ spreading matrix as $\mathbf{C}=\left[\mathbf{c}^{0} \mathbf{c}^{1} \ldots \mathbf{c}^{S-1}\right]$. The resulting spread symbols are added up and modulated, typically using the inverse fast Fourier transform (IFFT), onto the set of $N$ orthogonal subcarriers forming the group assigned to user $k$. A cyclic prefix $(\mathrm{CP})$ is appended to the resulting signal to minimise the effects of the channel dispersion. Assuming that the $\mathrm{CP}$ length exceeds the maximum channel delay spread, there will not be interference among successively transmitted blocks of symbols.

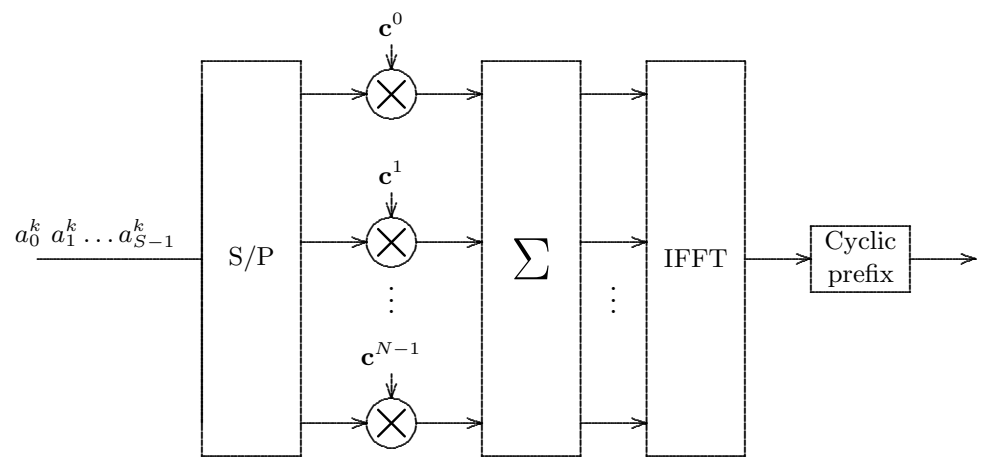

Fig. 1. GO-MC-CDM (group) transmitter

The signal from the base station reaches mobile user $k$ by propagating through a frequency selective channel with an scenario-dependent power delay profile $\mathcal{P}(\tau)$ given by

$$
\mathcal{P}(\tau)=\sum_{l=0}^{P-1} \phi(l) \delta\left(\tau-\tau_{l}\right)
$$




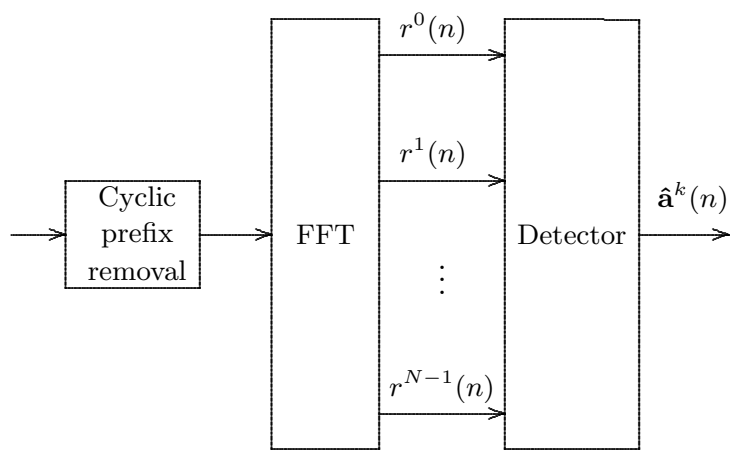

Fig. 2. GO-MC-CDM receiver

where $P$ denotes the number of independent paths of the channel and $\phi(l)$ and $\tau_{l}$ denote the power and delay of each path. It is assumed that the power delay profile is normalized to unity (i.e. $\sum_{l=1}^{l=P} \phi(l)=1$ ). A single realization of the channel impulse response from the base station to mobile user $k$ at time instant $t$ will then have the form

$$
h^{k}(t ; \tau)=\sum_{l=0}^{P-1} h_{l}^{k}(t) \delta\left(t-\tau_{l}\right)
$$

where it will hold that $E\left[\left|h_{l}^{k}(t)\right|^{2}\right]=\phi(l)$. The corresponding frequency response will be given by

$$
\bar{h}^{k}(t ; f)=\sum_{l=0}^{P-1} h_{l}^{k}(t) \exp \left(-j 2 \pi f \tau_{l}\right)
$$

which when evaluated at the $N$ subcarrier frequencies assigned to user $k$, yields the $N \times 1$ vector $\overline{\mathbf{h}}^{k}(t)=\left[\bar{h}^{k}\left(t ; f_{0}\right) \bar{h}^{k}\left(t ; f_{1}\right) \ldots \bar{h}^{k}\left(t ; f_{N-1}\right)\right]$. Assuming that the channel is static over the duration of a block of $S$ symbols and to simplify the notation, we will express the subcarrier frequency response for user $k$ during the discrete time instant $n$ as $\overline{\mathbf{h}}^{k}(n)=\left[\bar{h}_{0}^{k}(n) \bar{h}_{1}^{k}(n) \ldots \bar{h}_{N-1}^{k}(n)\right]$.

The reception process at the mobile receiver is illustrated in Fig. 2, After removing the cyclic prefix and assuming perfect subcarrier synchronization, the received signal is sampled and demodulated (tipically using the FFT) yielding the $N \times 1$ received signal vector which can be expressed as

$$
\mathbf{r}(n)=\mathbf{H}(n) \mathbf{C} \mathbf{a}^{k}(n)+\mathbf{v}(n)
$$

where $\mathbf{H}(n)=\mathcal{D}\left(\overline{\mathbf{h}}^{k}(n)\right)$ with $\mathcal{D}(\mathbf{x})$ denoting the squared diagonal matrix having vector $\mathbf{x}$ at its main diagonal. The $N \times S$ system matrix $\mathbf{A}(n)$ is defined as $\mathbf{A}(n)=\mathbf{H}(n) \mathbf{C}$ and represents the combination of channel and spreading effects. 
It can easily be seen that $\mathbf{A}(n)$ has the following structure

$$
\mathbf{A}(n)=\left(\begin{array}{ccc}
\bar{h}_{0}^{k}(n) c_{0}^{0} & \ldots & \bar{h}_{0}^{k}(n) c_{0}^{S-1} \\
\bar{h}_{1}^{k}(n) c_{1}^{0} & \ldots & \bar{h}_{1}^{k}(n) c_{1}^{S-1} \\
\vdots & \ddots & \vdots \\
\bar{h}_{N-1}^{k}(n) c_{N-1}^{0} & \ldots & \bar{h}_{N-1}^{k}(n) c_{N-1}^{S-1}
\end{array}\right)
$$

The $N \times 1$ complex vector $\mathbf{v}(n)$ is made of zero-mean complex Gaussian random variables with variance $E\left[v(n)^{2}\right]=\sigma_{v}^{2}$. Notice that with the definition of normalized unit-power transmitted symbols and normalized power delay profile, the operating signal-to-noise ratio can be expressed as $E_{s} / N_{0}=1 / \sigma_{v}^{2}$. In order to simplify the notation, and since successive symbols are independent from one another due to the $\mathrm{CP}$, the explicit time relation will be dropped from subsequent equations. Likewise and since the analysis focuses only on one group (i.e. user), the user index $k$ will also be dropped from now on.

At the mobile receiver, when using maximum likelihood multi-symbol detection (ML-MSD), the symbol estimates are computed according to [6]

$$
\hat{\mathbf{a}}=\underset{\mathbf{a}}{\operatorname{argmin}}\|\mathbf{A a}-\mathbf{r}\|^{2} .
$$

This procedure usually implies evaluating all the possible transmitted blocks of symbols and choosing the closest one (in a least-squares sense) to the received block. Recently, sphere detection [7] has been proposed for efficiently performing this search.

\section{Maximum Likelihood Detection Analysis}

The probability of symbol error when $S$ symbols are transmitted in a group can be upper bounded using the union bound as

$$
P_{s} \leq \frac{1}{S} \sum_{i=1}^{M^{S}} \sum_{j=1, j \neq i}^{M^{S}} P\left(\mathbf{a}_{i}\right) P\left(\mathbf{a}_{i} \rightarrow \mathbf{a}_{j}\right) \mathcal{N}_{s}\left(\mathbf{a}_{i}, \mathbf{a}_{j}\right)
$$

where $P\left(\mathbf{a}_{i}\right)$ is the probability of transmitting the $S \times 1$ block vector $\mathbf{a}_{i}$, $P\left(\mathbf{a}_{i} \rightarrow \mathbf{a}_{j}\right)$ represents the pairwise error probability (PEP) of erroneously detecting the $S \times 1$ block vector $\mathbf{a}_{j}$ and $\mathcal{N}_{s}\left(\mathbf{a}_{i}, \mathbf{a}_{j}\right)$ is the number of differing symbols between $\mathbf{a}_{i}$ and $\mathbf{a}_{j}$. Using the fact that all block vectors have the same probability of transmission, the probability of bit error can be upper bounded as

$$
P_{b} \leq \frac{1}{S M^{S} \log _{2} M} \sum_{i=1}^{M^{S}} \sum_{j=1, j \neq i}^{M^{S}} P\left(\mathbf{a}_{i} \rightarrow \mathbf{a}_{j}\right) \mathcal{N}_{b}\left(\mathbf{a}_{i}, \mathbf{a}_{j}\right)
$$

where $\mathcal{N}_{b}\left(\mathbf{a}_{i}, \mathbf{a}_{j}\right)$ is the number of differing bits between blocks $\mathbf{a}_{i}$ and $\mathbf{a}_{j}$.

To progress further in the analysis, the PEP conditioned on the system matrix $\mathbf{A}$ is first calculated. This can then be averaged with respect to $\mathbf{A}$ to yield the 
unconditional PEP. The PEP conditioned on a given system matrix $\mathbf{A}$ can be shown to be 8

$$
\begin{aligned}
P\left(\mathbf{a}_{i} \rightarrow \mathbf{a}_{j} \mid \mathbf{A}\right) & =\frac{1}{2} \operatorname{erfc}\left(\sqrt{\frac{\left\|\mathbf{A}\left(\mathbf{a}_{i}-\mathbf{a}_{j}\right)\right\|^{2}}{4 \sigma_{v}^{2}}}\right) \\
& =\frac{1}{\pi} \int_{0}^{\pi / 2} \exp \left(-\frac{\left\|\mathbf{A}\left(\mathbf{a}_{i}-\mathbf{a}_{j}\right)\right\|^{2}}{4 \sigma_{v}^{2} \sin ^{2} \phi}\right) d \phi
\end{aligned}
$$

We now define the error vector $\mathbf{e}_{i j}=\mathbf{a}_{i}-\mathbf{a}_{j}=\left[e_{i j}^{0}, e_{i j}^{1}, \ldots, e_{i j}^{S-1}\right]$ and the $N \times N$ diagonal matrix

$$
\mathbf{T}_{i j}=\left(\begin{array}{cccc}
\sum_{s=0}^{S-1} e_{i j}^{s} c_{0}^{s} & 0 & \cdots & 0 \\
0 & \sum_{s=0}^{S-1} e_{i j}^{s} c_{1}^{s} & \vdots \\
\vdots & & \ddots & 0 \\
0 & \ldots & 0 \sum_{s=0}^{S-1} e_{i j}^{s} c_{N-1}^{s}
\end{array}\right)
$$

We also define the $N \times N$ channel correlation matrix $\mathbf{R}=E\left[\overline{\mathbf{h}} \overline{\mathbf{h}}^{H}\right]$ which can be determined from the Fourier transform of $\mathcal{P}(\tau)$ (spaced-frequency correlation function) evaluated at the group-assigned subcarrier frequencies. It can be shown that $d_{i j}^{2} \triangleq\left\|\mathbf{A}\left(\mathbf{a}_{i}-\mathbf{a}_{j}\right)\right\|^{2}=\mathbf{e}_{i j}^{H} \mathbf{A}^{H} \mathbf{A} \mathbf{e}_{i j}=\overline{\mathbf{h}}^{H} \mathbf{T}_{i j}^{H} \mathbf{T}_{i j} \overline{\mathbf{h}}$ is a quadratic form in complex variable $\overline{\mathbf{h}}$ with moment generating function (MGF) 9 ]

$$
M_{d^{2}, i j}(w)=\left|\mathbf{I}+w \mathbf{T}_{i j} \mathbf{R} \mathbf{T}_{i j}^{H}\right|^{-1} .
$$

Let $\boldsymbol{\lambda}_{i j}=\left\{\lambda_{i j, 1}, \lambda_{i j, 2}, \ldots, \lambda_{i j, D_{i j}}\right\}$ denote the set of $D_{i j}$ distinct positive eigenvalues of $\mathbf{K}_{i j}=\mathbf{T}_{i j} \mathbf{R} \mathbf{T}_{i j}^{H}$ where the multiplicity of each eigenvalue is given by $\alpha_{i j, 1}, \alpha_{i j, 2}, \ldots, \alpha_{i j, D_{i j}}$. It is shown in [10] that the MGF of $d_{i j}^{2}$ can also be expressed as

$$
M_{d^{2}, i j}(w)=\prod_{d=1}^{D_{i j}} \frac{1}{\left(1+w \lambda_{i j, d}\right)^{\alpha_{i j, d}}}=\sum_{d=1}^{D_{i j}} \sum_{p=1}^{\alpha_{i j, d}} \frac{\kappa_{i j, d, p}}{\left(1+w \lambda_{i j, d}\right)^{p}}
$$

where

$$
\kappa_{i j, d, p}=\left.\frac{\lambda_{i j, d}^{p-\alpha_{i j, d}}}{\left(\alpha_{i j, d}-p\right) !} \frac{\partial^{\alpha_{i j, d}-p}}{\partial^{\alpha_{i j, d}-p} w}\left[\prod_{\begin{array}{c}
d^{\prime}=1 \\
d^{\prime} \neq d
\end{array}}^{D_{i j}} \frac{1}{\left(1+w \lambda_{i j, d^{\prime}}\right)^{\alpha_{i j, d^{\prime}}}}\right]\right|_{w=-\frac{1}{\lambda_{i j, d}}}
$$

which allows the unconditional PEP to be written as

$$
\begin{aligned}
P & \left(\mathbf{a}_{i} \rightarrow \mathbf{a}_{j}\right)=\frac{1}{\pi} \sum_{d=1}^{D_{i j}} \sum_{p=1}^{\alpha_{i j, d}} \kappa_{i j, d, p} \int_{0}^{\pi / 2}\left(\frac{\sin ^{2} \phi}{\sin ^{2} \phi+\frac{\lambda_{i j, d}}{4 \sigma_{v}^{2}}}\right)^{p} d \phi \\
= & \sum_{d=1}^{D_{i j}} \sum_{p=1}^{\alpha_{i j, d}} \kappa_{i j, d, p}\left(\frac{1-\Omega\left(\frac{\lambda_{i j, d}}{4 \sigma_{v}^{2}}\right)}{2}\right)^{p} \sum_{g=0}^{p-1}\left(\begin{array}{c}
p-1+g \\
g
\end{array}\right)\left(\frac{1+\Omega\left(\frac{\lambda_{i j, d}}{4 \sigma_{v}^{2}}\right)}{2}\right)^{g}
\end{aligned}
$$


with $\Omega(c)=\sqrt{c /(1+c)}$. By substituting (13) into (8), a closed-form BER upper bound for an arbitrary power delay profile is obtained. It is later shown that this bound is tight and accurately matches the simulation results.

Since there are many pairs $\left(\mathbf{a}_{i}, \mathbf{a}_{j}\right)$ giving exactly the same PEP, it is possible to define a pairwise error class $\mathcal{C}\left(\hat{D}_{c}, \hat{\boldsymbol{\lambda}}_{c}\right)$ as the set of all pairs $\left(\mathbf{a}_{i}, \mathbf{a}_{j}\right)$ characterized with a matrix $\mathbf{K}_{i j}$ with rank $\hat{D}_{c}$ and a set of eigenvalues $\hat{\boldsymbol{\lambda}}_{c}=$ $\left\{\hat{\lambda}_{c, 1}, \ldots, \hat{\lambda}_{c, \hat{D}_{c}}\right\}$ and therefore, a common PEP denoted by $\operatorname{PEP}\left(\hat{D}_{c}, \hat{\boldsymbol{\lambda}}_{c}\right)$. A more insightful BER expression (in comparison with (8) ) can then be obtained by using the PEP class notation, avoiding the exhaustive computation of all the PEPs. Instead, the BER upper-bound can be found by finding out the PEP for each class and weighing it using the number of elements in the class and the number of erroneous bits this class may induce. The BER upper bound can then be rewritten as

$$
P_{b} \leq \frac{1}{S M^{S} \log _{2} M} \sum_{\forall \mathcal{C}\left(\hat{D}_{c}, \hat{\boldsymbol{\lambda}}_{c}\right)} \sum_{i=1}^{S \log _{2} M} W\left(\hat{D}_{c}, \hat{\boldsymbol{\lambda}}_{c}, i\right) i \operatorname{PEP}\left(\hat{D}_{c}, \hat{\boldsymbol{\lambda}}_{c}\right)
$$

where $W\left(\hat{D}_{c}, \hat{\boldsymbol{\lambda}}_{c}, i\right)$ corresponds to the number of elements in the class $\mathcal{C}\left(\hat{D}_{c}, \hat{\boldsymbol{\lambda}}_{c}\right)$ inducing $i$ erroneous bits.

\section{Asymptotic Performance}

In order to gain further insight on the parameters affecting the BER in a GOMC-CDM system, we now focus on the asymptotic case of large $E_{s} / N_{0}$. It is easy to see that when $w \rightarrow \infty$ the MGF from (12) can be approximated by

$$
M_{d^{2}, i j}(w) \simeq \frac{1}{\left(\prod_{d=1}^{D_{i j}} \lambda_{i j, d}^{\alpha_{i j, d}}\right) w^{\sum_{d=1}^{D_{i j}} \alpha_{i j, d}}}
$$

allowing $\operatorname{PEP}\left(\hat{D}_{c}, \hat{\boldsymbol{\lambda}}_{c}\right)$ for large $E_{s} / N_{0}$ to be expressed as

$$
\begin{aligned}
& \operatorname{PEP}_{\text {asym }}\left(\hat{D}_{c}, \hat{\boldsymbol{\lambda}}_{c}\right)=\frac{1}{\pi} \int_{0}^{\pi / 2} \frac{\left(4 \sigma_{v}^{2} \sin ^{2} \phi\right)^{\hat{D}_{c}}}{\prod_{d=1}^{\hat{D}_{c}} \hat{\lambda}_{c, d}^{\hat{\alpha}_{c, d}}} d \phi \\
& =\frac{\Gamma\left(\hat{D}_{c}+\frac{1}{2}\right)}{2 \sqrt{\pi} \Gamma\left(\hat{D}_{c}+1\right)} \frac{\left(4 \sigma_{v}^{2}\right)^{\hat{D}_{c}}}{\prod_{d=1}^{\hat{D}_{c}} \hat{\lambda}_{c, d}^{\hat{\alpha}_{c, d}}}=\frac{\left(2 \hat{D}_{c}\right) !}{2 \hat{D}_{c} !^{2}} \frac{\left(E_{s} / N_{0}\right)^{-\hat{D}_{c}}}{\prod_{d=1}^{\hat{D}_{c}} \hat{\lambda}_{c, d}^{\hat{\alpha}_{c, d}}}
\end{aligned}
$$

where $\Gamma(x)$ denotes the Gamma function. Equation (16) implies that, asymptotically, the dominant terms in (14) are those corresponding to pairwise error classes associated with $\mathbf{K}$-matrices of minimum rank, that is, $\hat{D}_{c}=\hat{D}_{\min }$, allowing the BER to be asymptotically approximated by

$$
P_{b} \leq \frac{1}{S M^{S} \log _{2} M} \sum_{\forall \mathcal{C}\left(\hat{D}_{m i n}, \hat{\boldsymbol{\lambda}}_{c}\right)} \sum_{i=1}^{S \log _{2} M} i \frac{\left(2 \hat{D}_{\min }\right) !}{2\left(\hat{D}_{\min } !\right)^{2}} \frac{W\left(\hat{D}_{m i n}, \hat{\boldsymbol{\lambda}}_{c}, i\right)\left(\frac{E_{s}}{N_{0}}\right)^{-\hat{D}_{\text {min }}}}{\prod_{d=1}^{\hat{D}_{m i n}} \hat{\lambda}_{c, d}^{\hat{\alpha}_{c, d}}}
$$


This expression indicates that BER can be minimized by simultaneously maximizing $\hat{D}_{\text {min }}$ and $\prod_{d=1}^{\hat{D}_{\text {min }}} \hat{\lambda}_{c, d}^{\hat{\alpha}_{c, d}}$, therefore it is important to carefully perform the subcarrier allocation and the selection of the family of spreading codes with these objectives in mind. As it happens in the uplink 4, choosing the subcarriers for a group equispaced across the whole bandwidth minimizes subcarrier correlation allowing us to optimize the system performance if an adequate family of codes is properly selected. To this end, rotated spreading transforms have been proposed for downlink multicarrier systems [1].

\section{$5 \quad$ Rotated Spreading}

It is shown in 11] that the often used Walsh-Hadamard codes lead to poor diversity gains when employed to perform the frequency spreading in the downlink of multicarrier systems. This can be explained by the fact that for certain symbol blocks, the energy is concentrated on one single subcarrier. A deep fade on this subcarrier dramatically raises the probability of error in the detection process, irrespective of the state of all other subcarriers, limiting in this way the achievable diversity order (asymptotic BER slope) to one. A similar effect is observed in the GO-MC-CDM framework under study which is best illustrated through an example: suppose 4 subcarriers are used to transmit 4 symbols multiplexed by code using binary modulation with alphabet $\{+1,-1\}$ and Walsh-Hadamard spreading. In order to find the BER upper-bound, the different pairwise error classes need to be computed as indicated by (14). One of these classes will comprise the PEP between blocks which differ in all symbols such as $a_{i}=\left[\begin{array}{llll}1 & 1 & 1 & 1\end{array}\right]^{T}$

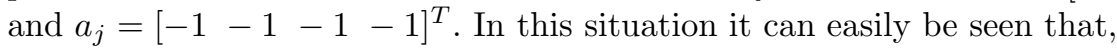

$$
\mathbf{T}_{i, j}=\left(\begin{array}{cccc}
8 & 0 & 0 & 0 \\
0 & 0 & 0 & 0 \\
0 & 0 & 0 & 0 \\
0 & 0 & 0 & 0
\end{array}\right), \quad \mathbf{K}_{i, j}=\left(\begin{array}{ccccc}
8 R_{0,0} & 0 & 0 & 0 \\
8 R_{1,0} & 0 & 0 & 0 \\
8 R_{2,0} & 0 & 0 & 0 \\
8 R_{3,0} & 0 & 0 & 0
\end{array}\right)
$$

where $R_{x, y}$ denotes the $(x, y)$ th entry of the channel correlation matrix. Obviously, for this particular PEP, $\hat{D}_{\min }=1$ and this will become the dominant term in the BER expression given by (14) leading to a diversity order of one. For the particular case of Walsh-Hadamard sequences, this behavior is due to the fact that all columns (or rows) add up to zero except for the first entry. A similar effect can be observed in other spreading sequences such as those based on the discrete Fourier transform (DFT). As pointed out in [11, a spreading that maximizes the diversity gain can be found by applying a rotation to the columns of the conventional spreading matrix $\mathbf{C}$

$$
\mathbf{C}_{\text {rot }}=\mathbf{C D}(\boldsymbol{\theta})
$$

where $\boldsymbol{\theta}=\left[\theta_{0} \theta_{1} \ldots \theta_{N-1}\right]$ with each $\theta_{i}$ denoting the chip-specific rotation which in the scheme proposed in [1] is given by

$$
\theta_{i}=\exp \left(\frac{2 \pi j c_{i}^{s}}{N \Delta}\right) \quad s=0,1, \ldots, N-1 .
$$


with $\Delta$ being constellation dependent and is selected so as to make $2 \pi / \Delta$ the minimum angle which rotates the transmit symbol alphabet onto itself $(\Delta=2$ for the case of BPSK). Table 5 lists the characteristics of the different pairwise error classes for Walsh-Hadamard spreading and its rotated version for a group size of four BPSK symbols $(S=4)$. In contrast to conventional Walsh-Hadamard which attains $\hat{D}_{\min }=1$, for rotated Walsh-Hadamard spreading, all classes are characterized by $\hat{D}_{c}=\hat{D}_{\text {min }}=4$. Taking into account the asymptotic BER expression in (17), this indicates that while using conventional Walsh-Hadamard spreading no diversity gain will be achieved, the rotated spreading has the potential (depending on the channel correlation matrix $R$ ) to attain a diversity gain equal to the number of employed subcarriers. Notice that, logically, in both cases there are the same number of different pairwise errors $\left(240=4^{2} \times 4^{2}-4^{2}\right)$. We conclude this section by remarking that here, only the maximization $\hat{D}_{\text {min }}$ has been pursued. Maximization of the product of eigenvalues $\left\{\hat{\lambda}_{\hat{D}_{\text {min }}, d}\right\}_{d=1}^{\hat{D}_{\text {min }}}$ is a topic of current research.

Table 1. Ranks and number of entries of the different PEP classes

\begin{tabular}{|c|c|c|c|c|c|c|c|}
\hline \multicolumn{4}{|c|}{ Walsh-Hadamard } & \multicolumn{4}{|c|}{ Rotated Walsh-Had. } \\
\hline$W\left(\hat{D}_{c}, \hat{\boldsymbol{\lambda}}_{c}, i\right)$ & $\hat{D}_{c}$ & $i$ & $\hat{\boldsymbol{\lambda}}_{c}$ & $W\left(\hat{D}_{c}, \hat{\boldsymbol{\lambda}}_{c}, i\right)$ & $\hat{D}_{c}$ & $i$ & $\hat{\boldsymbol{\lambda}}_{c}$ \\
\hline 8 & 1 & 4 & {$[64]$} & 64 & 4 & 1 & {$\left[\begin{array}{llll}3.29 & 3.39 & 4.25 & 5.07\end{array}\right]$} \\
\hline 32 & 2 & 2 & {$\left[\begin{array}{lll}15.09 & 16.91\end{array}\right]$} & 32 & 4 & 2 & {$\left[\begin{array}{llll}10.13 & 8.51 & 6.58 & 6.78\end{array}\right]$} \\
\hline 64 & 2 & 2 & {$\left[\begin{array}{lll}14.06 & 17.93\end{array}\right]$} & 64 & 4 & 2 & {$\left[\begin{array}{llll}2.17 & 2.36 & 14.5 & 12.92\end{array}\right]$} \\
\hline 64 & 4 & 1 & {$\left[\begin{array}{llll}3.29 & 3.39 & 4.25 & 5.07\end{array}\right]$} & 64 & 4 & 3 & {$\left[\begin{array}{lllll}23.78 & 13.14 & 10.42 & 0.66\end{array}\right]$} \\
\hline 64 & 4 & 3 & {$\left[\begin{array}{lllll}3.33 & 3.81 & 4.7 & 36.14\end{array}\right]$} & 4 & 16 & 4 & {$\left[\begin{array}{llll}30.74 & 24.8 & 5.11 & 4.06\end{array}\right]$} \\
\hline 8 & 4 & 4 & {$\left[\begin{array}{llll}13.16 & 13.55 & 17.01 & 20.24\end{array}\right]$} & & & & \\
\hline$\Sigma=240$ & & & & $\Sigma=240$ & & & \\
\hline
\end{tabular}

\section{$6 \quad$ Numerical Results}

We assume a system operating around $5 \mathrm{GHz}$ with a total bandwidth of $100 \mathrm{MHz}$, such parameters are typically used in $4 \mathrm{G}$ systems currently under discussion (see for example [12]). The number of total subcarriers, $N_{\text {total }}$, is fixed to 2048 ensuring that each subcarrier undergoes frequency flat fading. The power delay profile of the ETSI BRAN channel A (typical office environment) has been used. This channel has 18 independent Rayleigh fading paths with an rms delay spread of $50 \mathrm{~ns}$.

Figure 3 shows the simulation (circles) and analytical results (solid lines) when using different number of subcarriers in combination with conventional WalshHadamard spreading. It is seen how the theoretical upper-bound provides an accurate approximation of the true BER for $E_{s} / N_{0}$ ratios of practical interest. Notice that the more subcarriers being used, the looser the bound is for low $E_{s} / N_{0}$ levels. This behavior is typical in analysis based on the union bound. 


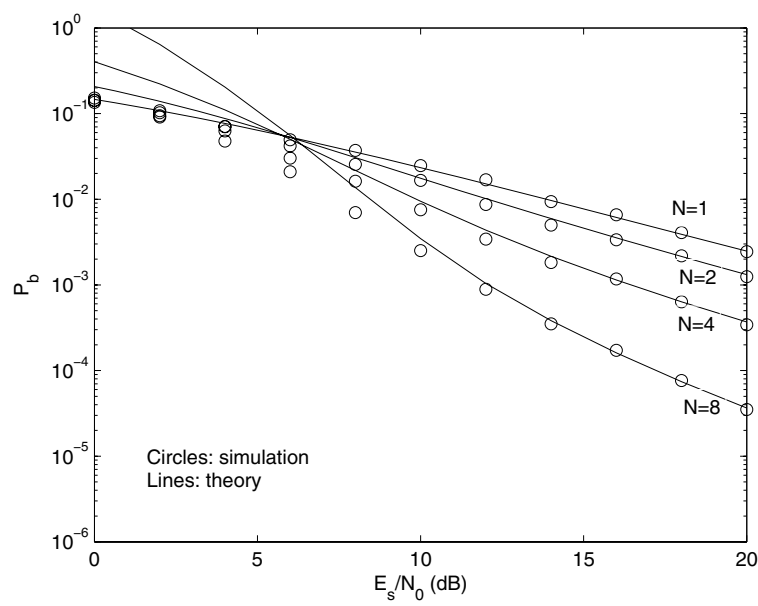

Fig. 3. BER when using $\mathrm{N}=1,2,4$ and 8 subcarriers. Full load $(N=S)$. WalshHadamard spreading. BPSK modulation.

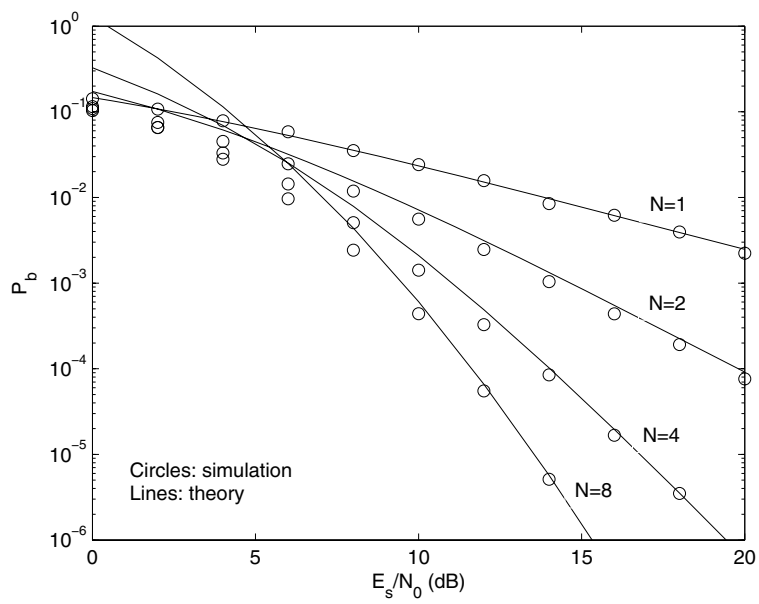

Fig. 4. BER when using $N=1,2,4$ and 8 subcarriers. Full load $(N=S)$. Rotated Walsh-Hadamard spreading. BPSK modulation.

It can also be seen that, when using conventional Walsh-Hadamard spreading, increasing the number of subcarriers per group brings along a reduction in BER, however, it is already hinted in this figure that the asymptotic slope of the BER curves when using different number of subcarriers is the same. It has been shown in Section 5 that Walsh-Hadamard codes of length 4 induce $\hat{D}_{\min }=1$. Indeed this is also the case for any other code length. It is seen in Fig. 3 that, as predicted by the asymptotic analysis in Section 4, all curves tend to have a asymptotic slope of one (for $N=8$ this holds at around $E_{s} / N_{0}=40 \mathrm{~dB}$ ). 
Figure 4 presents the same set of simulations but now using rotated WalshHadamard spreading. Again, good agreement can be observed between simulation (circles) and analytical (solid lines) results. The improvement with respect to conventional spreading is very clear. As an example, for $N=4$, the rotated spreading provides a $4 \mathrm{~dB}$ gain with respect to the non-rotated one for $P_{b}=10^{-3}$, for lower $P_{b}$ 's gains are even larger. It was shown in Section 5 that with the rotated spreading and $N=4$, all pairwise error classes had $\hat{D}_{c}=\hat{D}_{\text {min }}=4$ maximizing the achievable diversity order for a given channel correlation matrix and a specific number of subcarriers per group. It can indeed be seen in Fig. 4 that the BER curve for $N=4$, at large $E_{s} / N_{0}$ levels, has a diversity order equal to four in agreement with the asymptotic prediction.

\section{Conclusions}

A new scheme, which we call group-orthogonal multicarrier code-division multiplex (GO-MC-CDM), suitable for the downlink segment of fourth generation of wireless communication systems has been proposed. This scheme allows the efficient use of multi-symbol detection schemes and offers the same performance as (downlink) GO-MC-CDMA at a fraction of its computational cost. The performance of this system has been analyzed when using maximum likelihood multi-symbol detection. A closed-form BER expression has been derived which reveals which are the important parameters to be taken into account in order to optimize the BER performance. In particular, choosing the subcarriers for a group to be equispaced over the whole available bandwidth and selecting a family of rotated spreading codes prove to be of fundamental importance in the minimization of the BER. Simulation results have also been presented validating the analytical derivations.

\section{Acknowledgments}

This work has been supported in part by the MEC and FEDER under project MARIMBA (TEC2005-0997) and a Ramon y Cajal fellowship, Spain.

\section{References}

1. N. Yee, J.-P. Linnartz, and G. Fettweis, "Multi-carrier CDMA in indoor wireless radio networks," in Proc. IEEE Int. Symp. on Pers., Indoor and Mob. Rad. Comm., Yokohama (Japan), Sept. 1993, pp. 109-113.

2. S. Hara and R. Prasad, "Overview of multicarrier CDMA," IEEE Communications Mag., vol. 35, pp. 126-133, December 1997.

3. K. Fazel and S. Kaiser, Multi-Carrier and Spread Spectrum Systems. Wiley, 2003.

4. X. Cai, S. Zhou, and G. Giannakis, "Group-orthogonal multicarrier CDMA," IEEE Trans. Communications, vol. 52, no. 1, pp. 90-99, January 2004.

5. S. Kaiser, "OFDM code-division multiplexing in fading channels," IEEE Trans. Communications, vol. 50, pp. 1266-1273, 2002. 
6. J. Proakis, Digital Communications, 3rd ed. Mc-Graw Hill, 1996.

7. B. Hochwald and S. ten Brink, "Achieving near-capacity on a multiple-antenna channel," IEEE Trans. Communications, vol. 51, pp. 389-399, 2003.

8. J. W. Craig, "A new, simple and exact result for calculating the probability of error for two-dimensional signal constellations," in IEEE MILCOM'91 Conf. Rec., Boston, MA, 1991, pp. 25.5.1-25.5.5.

9. M. Schwartz, W. Bennett, and S. Stein, Communications Systems and Techniques. Wiley-IEEE Press, 1995.

10. G. Femenias, "BER performance of linear STBC from orthogonal designs over MIMO correlated nakagami-m fading channels," IEEE Trans. Vehicular Tech., vol. 53, pp. 307-317, 2004.

11. A. Bury, J. Egle, and J. Lindner, "Diversity comparison of spreading transforms for multicarrier spread spectrum transmission," IEEE Trans. Communications, vol. 51, no. 5, pp. 774-781, May 2003.

12. "European union framework program 6 - WINNER project," https://www.istwinner.org/. 